\title{
Morbimortalidad en la endarterectomía carotídea
}

\author{
J.M. Castilla; P. Delgado; V. Martín; O. Fernández-Arconada; M.A. Martín-Santidrián y A. Rodríguez-Salazar
}

Servicio de Neurocirugía. Hospital General Yagüe. Burgos.

Resumen

Objetivo. Evaluar las complicaciones perioperatorias y a largo plazo en pacientes tratados mediante endarterectomía por estenosis carotídea en nuestro Servicio.

Pacientes y métodos. Estudio retrospectivo de 97 endarterectomías realizadas por 6 neurocirujanos en 90 pacientes entre enero de 1995 y diciembre de 2003. Diez de los pacientes fueron mujeres. La mediana de edad fue 69 años (rango 38-86). Siete pacientes se intervinieron bilateralmente. Ochenta y cuatro estenosis fueron mayores del $70 \%$. El número de cirugías anual por cirujano fue de 3 (rango 0-10). El seguimiento clínico fue de 121 días, (rango 8-2106).

Resultados. Fallecieron 4 pacientes en el postoperatorio inmediato y otros 4 presentaron déficits neurológicos añadidos, con una morbimortalidad de $\mathbf{8}^{\prime} \mathbf{9} \%$ de los pacientes y $\mathbf{8}^{\prime} \mathbf{2} \%$ de las cirugías. Cuatro pacientes se reintervinieron, 1 por déficit neurológico inmediato y 3 por hematoma quirúrgico. Hubo afectación transitoria de nervio periférico en 8 pacientes y se registraron 21 complicaciones médicas asociadas. Posteriormente 7 pacientes fallecieron (6 por cáncer y 1 por cardiopatía) y 5 presentaron ictus ( 3 ipsilaterales y 2 de otra localización). En el último control postoperatorio por imagen, a los 52 (0-2832) días, se constataron 7 estenosis en la arteria operada.

Conclusiones. La endarterectomía carotídea puede realizarse con razonable seguridad en centros con bajo volumen de pacientes, con métodos sencillos y con resultados aceptables. El conocimiento de los resultados reales obtenidos en cada centro es importante para mejorarlos.

PALABRAS CLAVE: Endarterectomía carotídea. Complicaciones. Mortalidad. Control de calidad. Evaluación de riesgos. Selección de pacientes.

Morbimortality in carotid endarterectomy

Recibido: 27-05-04. Aceptado: 18-08-04
Summary

Objective. To assess perioperative and long-terco morbidity in patients diagnosed of carotid stenosis submitted to our Department for surgical endarterectomy.

Patients and methods. A retrospective study of 97 endarterectomies performed by six neurosurgeons in 90 patients treated between january 1995 and december 2003. Ten patients were women. Average mean age was 69 years-old (range $38-86$ ). Seven patients were treated bilateraly. Eighty-four stenosis were greater than $\mathbf{7 0} \%$. Annual number of interventions per surgeon was 3 (range 0-10). The median follow-up was 121 days, (range 8-2106).

Results. Four patients died perioperatively and another 4 developed new neurologic deficits. The combined morbiditymortality rate was $8^{\prime} 9 \%$ of the patients and $8.2 \%$ of the surgeries. Four patients needed reintervention, because of immediate postoperative new deficit (one) and surgical hematoma (three). Transient peripheral nerve palsy occurred in 8 patients and 21 medical complications were registered. In the long term, 7 patients died ( 6 because of cancer and 1 because of cardiopathy) and 5 presented neurologic events (3 ipsilateral and 2 in other locations). Last postoperative image control, performed on average after 52 days (02832), revealed 7 estenosis of the treated artery.

Conclusions. Carotid endarterectomy can be safely performed in low-volume centers with acceptable results and reasonable morbidity and mortality rates when simple techniques are used. We consider crucial to evaluate self complications and results in order to improve them.

KEY WORDS. Carotid endarterectomy. Complications. Mortality. Quality control. Risk assessment. Patient selection.

Abreviaturas. CASANOVA: Carotid Artery Stenosis with Asymptomatic Narrowing: Operation VS Aspirin. ECST: European Carotid Surgical Trial. EEG: Electro EncefaloGrama. NASCET: North American Symptomatic Carotid Endarterectomy Trial. RM: Resonancia Magnética. TAC: Tomografia Axial Computarizada. 


\section{Introducción}

La enfermedad carotídea se considera responsable de, al menos, $20-30 \%$ de los ictus cerebrales ${ }^{11,26,67}$. Estos suponen la tercera causa de muerte en los países industrializa$\operatorname{dos}^{43}$. La limitación que padecen los enfermos con secuelas neurológicas postictales altera significativamente su calidad de vida y su entorno socio-familiar. El envejecimiento progresivo de la población hace el problema más significativo, pues la prevalencia de estenosis carotídea asintomática en la población oscila entre 0 '5 y $10 \%$ a medida que aumenta la edad del sujeto ${ }^{7}$ y la incidencia y prevalencia de ictus aumenta también con la edad, dándose el $80 \%$ de ellos en mayores de 65 años $^{37}$.

Varios estudios han demostrado la efectividad de la endarterectomía carotídea para prevenir infartos cerebrales en pacientes sintomáticos con moderado-alto grado de estenosis. La morbimortalidad quirúrgica, en relación con las características del paciente y del cirujano es un factor importante a tener en cuenta respecto a la decisión terapéutica $^{16,24,27,46,50,53,56,62}$. Las indicaciones quirúrgicas se van extendiendo de nuevo cada vez más, incluso para pacientes de alto riesgo quirúrgico o ancianos ${ }^{37,55}$, a veces excediendo las recomendaciones establecidas, especialmente en pacientes asintomáticos de alto riesgo ${ }^{35}$.

La terapia endovascular con eficacia y morbilidad mayor similares, permite evitar complicaciones menores asociadas a la cirugía ${ }^{14}$. Generalmente aún no se considera un tratamiento plenamente equiparable al quirúrgico $^{14,19,40,44,47}$, y se recomienda reservarlo para los pacientes en quienes el riesgo de una endarterectomía es prohibitivo clínica o técnicamente ${ }^{30,44}$.

No basta con extrapolar los resultados de los estudios randomizados a todos los pacientes y cirujanos, sino que es necesaria una adecuada selección de los pacientes a tratar. Se deben conocer además las características de cada centro en particular para una decisión individualizada, realizando auditorías periódicas ${ }^{29,41,48,56}$. El presente estudio pretende aclarar los resultados de la endarterectomía quirúrgica en nuestro Servicio, y lo que realmente podemos ofrecer a los pacientes, presentando nuestra experiencia con dicha técnica desde el punto de vista de la morbimortalidad.

\section{Pacientes y métodos}

Se analizan retrospectivamente las historias clínicas de los pacientes intervenidos mediante endarterectomía carotídea en el Servicio de Neurocirugía del Hospital "General Yagüe" entre enero de 1995 y diciembre de 2003. Los pacientes fueron remitidos por la Sección de Neurología, tras haber presentado sintomatología correspondiente con la arteria estenosada.

La técnica quirúrgica que se empleó corresponde a las descripciones habituales ${ }^{12,20,45}$ (Figura 1). En resumen, bajo anestesia general (salvo 4 casos), se abre longitudinalmente la piel y el platisma y se diseca anteriormente al músculo esternocleidomastoideo, dividiendo la vena facial y separando la vena yugular interna lateralmente. Se aislan la arteria carótida común y sus ramas del conectivo adyacente. Se clipan los vasos (arteria tiroidea, carótida interna, común y externa, en ese orden) tras heparinización sistémica y con elevación previa de la tensión arterial, sin depresión barbitúrica ni monitorización neurológica, y se abre la arteria. La placa se diseca preferiblemente en una pieza, y se retiran exhaustivamente sus restos. Los vasos se desclampan temporal e individualmente para facilitar la salida de restos de placa y luego se retira el clampaje en orden inverso al de colocación. Se emplea una sutura simple sin parche para el cierre. La heparinización se revierte con protamina y se cierra la herida tras una cuidadosa hemostasia, dejando un drenaje aspirativo. El paciente se despierta en quirófano. Luego pasa a la unidad de recuperación post-anestésica o unidad de cuidados intensivos y posteriormente a la planta.

El diagnóstico y grado de estenosis carotídea, se hizo con eco-Doppler y se confirmó mediante angiografía. La angio-TAC y angio-RM se emplearon ocasionalmente (Figura 2). Se clasificaron a los pacientes en varias categorías: menores del $70 \%(<70 \%)$, entre el 70 y $79 \%$, entre el 80 y $89 \%$, entre 90 y $99 \%$, y aquellas con obstrucción completa del flujo. Las diferencias entre las mediciones obtenidas mediante eco-Doppler y las obtenidas mediante angiografía fueron estudiadas mediante pruebas estadísticas de contraste de hipótesis, asumiendo que se trataba de datos categóricos ordenados mediante una escala ordinal, y que los intervalos de la escala representan diferencias iguales en los estadísticos que calculamos ${ }^{22}$.

El seguimiento se realizó en la consulta externa de Neurocirugía, y consistió en una valoración clínica y un control eco-Doppler unas semanas tras la intervención en la mayoría de los pacientes y posteriormente según evolución. También se han recogido datos pertinentes de otras consultas o ingresos cuando se dispuso de ellos.

Se describen los casos de éxitus perioperatorios y tardíos, complicaciones neurológicas inmediatas y a largo plazo, complicaciones de la cirugía que requirieron reintervención o alargaron el tratamiento, reestenosis y otras morbilidades

\section{Resultados}

\section{Pacientes y procedimientos}

Entre enero de 1995 y diciembre de 2003 se practicaron en nuestro servicio 97 intervenciones en 90 pacientes $(80$ varones y 10 mujeres) con estenosis carotídea. La mediana 

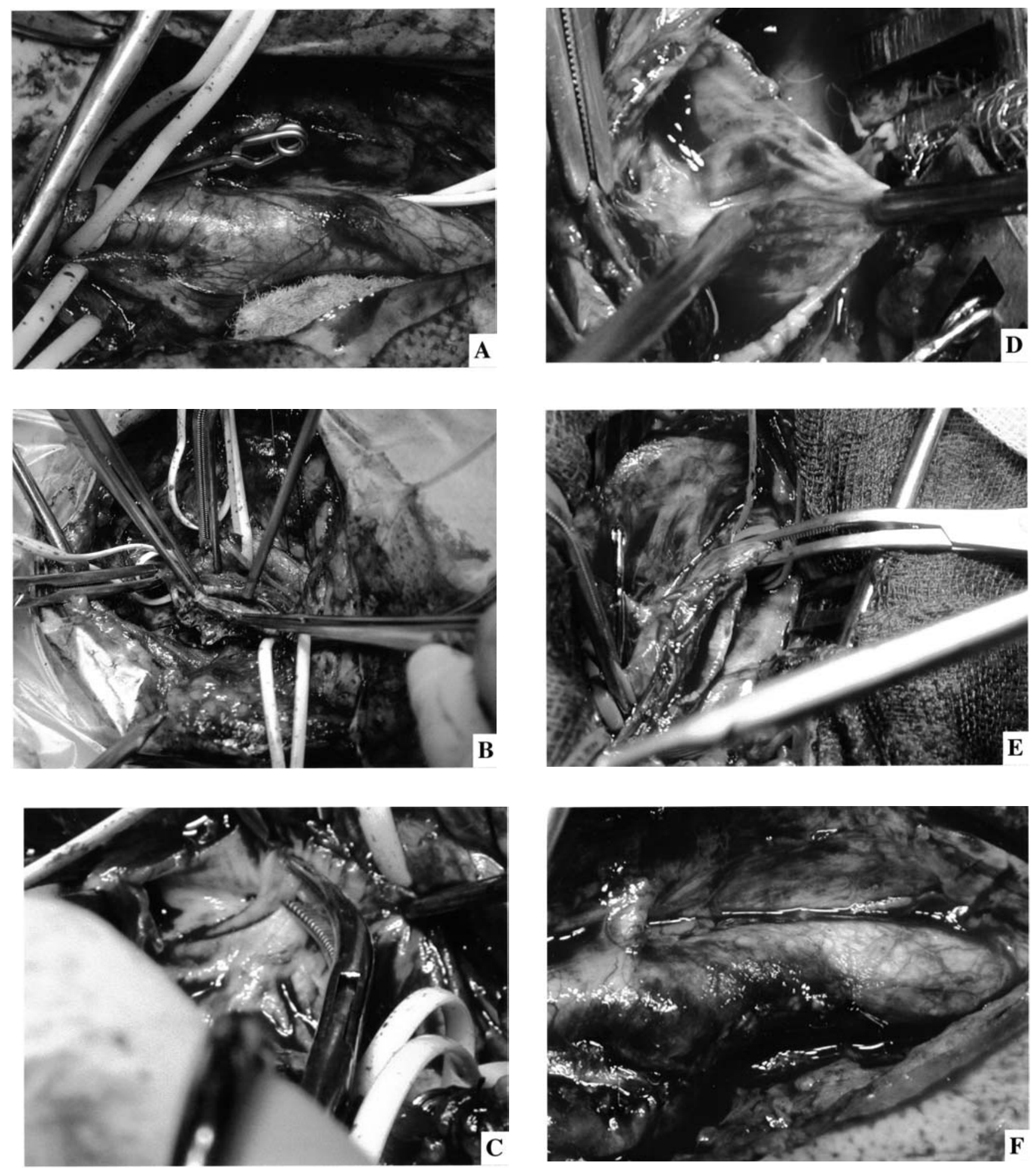

Figura 1. A: Exposición de una bifurcación carotídea derecha antes del clampaje y arteriotomía. Se ha colocado un clip en la arteria tiroidea y se han rodeado las carótidas común, interna y externa con sendas cintas.

$B, C, D, E$ : Tras clampar los vasos y abrir la pared arterial, se elimina progresivamente la placa ateromatosa y sus restos. F: Aspecto final de la arteriotomía suturada. 

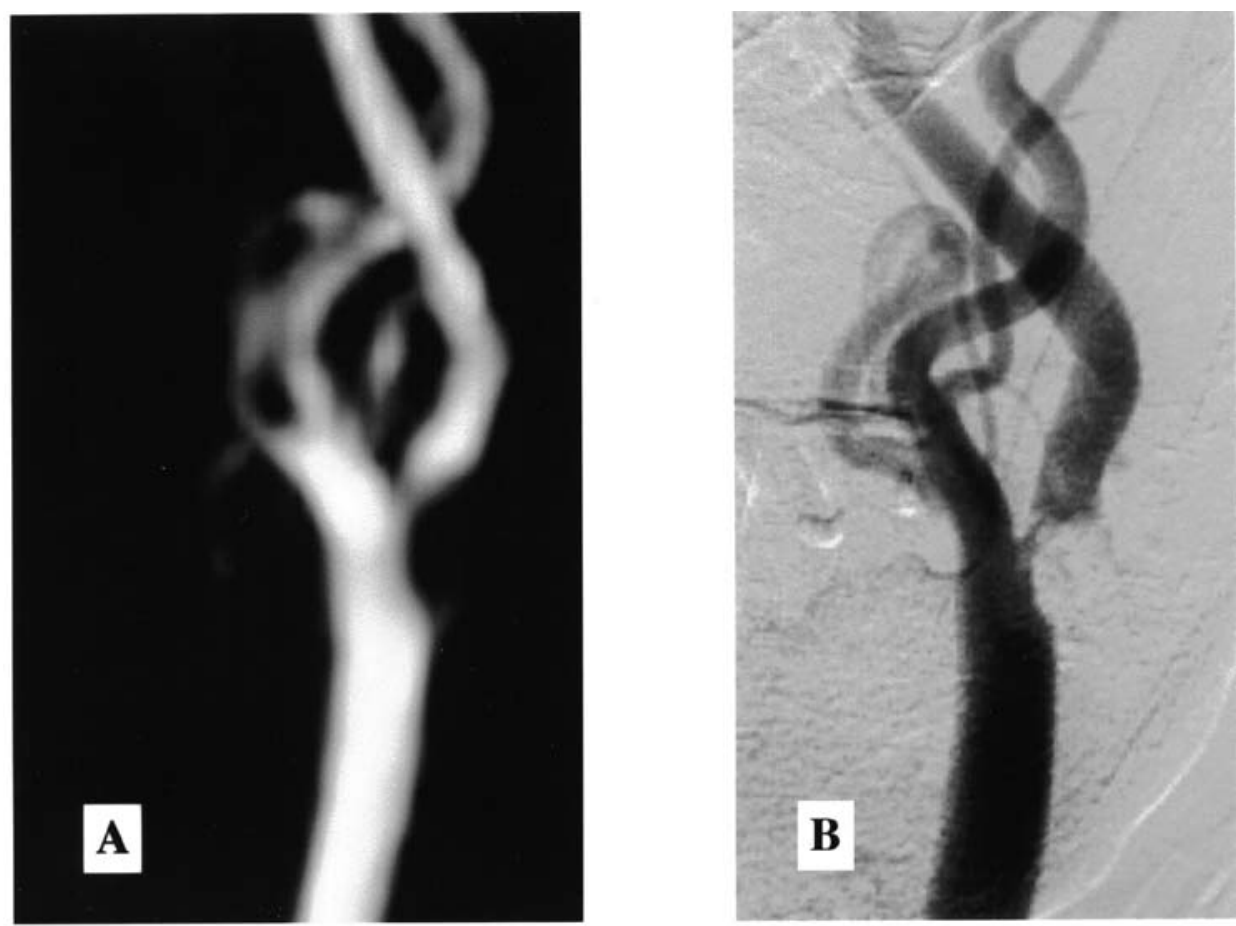

Figura 2. Imagen de la estenosis de la arteria carótida interna de un paciente mediante angio-resonancia (A) y angiografia digital convencional (B). de edad fue de 69 años, rango 38-86. Nueve de los pacientes tenían 80 o más años y 11 de ellos menos de 60 años en el momento de la cirugía. Se intervinieron 47 carótidas derechas y 42 izquierdas. Se realizaron una mediana de 10 cirugías por año, rango 8-15. Cada cirujano realizó entre 4 y 46 intervenciones en el período estudiado, con una mediana anual de 3, rango 0-10.

Las características preoperatorias de los pacientes según varias escalas se muestran en la Tabla 1 y los antecedentes clínicos en la Tabla 2.

Once pacientes fueron operados bilateralmente (Tabla 3). Se han excluido de la consideración del presente estudio cuatro de sus intervenciones: una paciente (rr) con una obstrucción completa de la carótida interna que se rellenaba retrógradamente a través de la externa y que le producía clínica ipsilateral, se trató mediante una endarterectomía carotídea externa sin complicaciones perioperatorias. Posteriormente fue intervenida de una estenosis progresiva de la carótida interna del otro lado. Sólo se ha considerado en el presente análisis la segunda cirugía. Otras tres endarterectomías practicadas en dos varones y una mujer por el servicio de Cirugía Vascular (CV) 3 años antes, 2 meses y 2 años después de nuestros procedimientos tampoco han sido incluidas en el presente estudio.

Tabla 1

Datos clínicos preoperatorios (*)

\begin{tabular}{|c|c|c|c|c|c|}
\hline $\boldsymbol{A S A}$ & $\boldsymbol{I}$ & $\boldsymbol{I I}$ & $\boldsymbol{I I I}$ & $\boldsymbol{I}$ & \multicolumn{1}{|c|}{} \\
\hline & - & 48 & 43 & 5 & - \\
\hline
\end{tabular}

\begin{tabular}{|l|c|c|c|c|c|c|c|}
\hline Rankin & $\boldsymbol{0}$ & $\boldsymbol{I}$ & $\boldsymbol{I I}$ & $\boldsymbol{I I I}$ & $\boldsymbol{I V}$ & $\boldsymbol{V}$ \\
\hline & 43 & 16 & 21 & 13 & 3 & - \\
\hline
\end{tabular}

\begin{tabular}{|c|c|c|c|c|c|c|c|c|c|c|c|}
\hline Can & $\mathbf{1 0 0}$ & $\mathbf{9 5}$ & $\mathbf{9 0}$ & $\mathbf{8 5}$ & $\mathbf{8 0}$ & $\mathbf{7 5}$ & $\mathbf{7 0}$ & $\mathbf{6 0}$ & $\mathbf{5 5}$ & $\mathbf{3 0}$ & $\mathbf{2 5}$ \\
\hline & 48 & 16 & 7 & 6 & 4 & 6 & 1 & 2 & 3 & 2 & 1 \\
\hline
\end{tabular}

\footnotetext{
* en un paciente con datos incompletos no se puede determinar su clasificación. Rankin (escala de Rankin modificada). $\boldsymbol{A S \boldsymbol { A }}$ (escala de gradación de riesgo anestésico de la American Society of Anestbesiologists). Can (escala neurológica canadiense).
} 
Tabla 2

Antecedentes clínicos

\begin{tabular}{|c|c|c|c|}
\hline Antecedentes por pacientes & todos & Operados de un lado & Operados bilateralmente \\
\hline Infarto cerebral & 49 & 45 & 4 \\
\hline Isquemia temporal & 41 & 37 & 4 \\
\hline hipertensión arterial & 39 & 35 & 4 \\
\hline hiperglucemia & 25 & 22 & 3 \\
\hline hiperlipidemias & 34 & 31 & 3 \\
\hline patología pulmonar & 18 & 17 & 1 \\
\hline \multicolumn{4}{|l|}{ cardiopatía } \\
\hline - enfermedad coronaria & 12 & 12 & 0 \\
\hline - infarto miocardio & 16 & 14 & 2 \\
\hline - angor & 11 & 9 & 2 \\
\hline - insuficiencia congestiva & 1 & 1 & 0 \\
\hline - arritmia & 14 & 12 & 2 \\
\hline - valvulopatía & 4 & 2 & 2 \\
\hline vasculopatía periférica & 20 & 17 & 3 \\
\hline cirugías previas & 41 & 36 & 5 \\
\hline Otras patologías asociadas previas & 27 & 23 & 4 \\
\hline tabaco & 48 & 41 & 7 \\
\hline alcohol & 32 & 28 & 4 \\
\hline obesidad & 9 & 9 & 0 \\
\hline
\end{tabular}

La Sección de Neurología estudió y siguió a los pacientes clínicamente y en su laboratorio de ultrasonografía, remitiéndonos los pacientes con sintomatología correspondiente con una arteria estenosada más del $70 \%$ o con una placa irregular y ulcerada. La sintomatología se había resuelto completamente en el momento de la cirugía en 45 casos, pero 51 pacientes se operaron con secuelas en grado variable en el momento de la cirugía; cuatro de estos pacientes presentaban afectación ocular, no reflejada en la escala canadiense (Tabla 1). De los operados bilateralmente, uno tenía sintomatología bilateral; los otros 7, con sintomatología debida a la carótida operada inicialmente, se intervinieron de la arteria contralateral por presentar en ella una estenosis significativa o una placa irregular. No se excluyeron pacientes por motivos clínicos o de edad, siempre que aceptaran los riesgos de la intervención.

El diagnóstico y grado de estenosis carotídea, se hizo mediante ecografía en todos los casos, aunque en tres pacientes el estudio no fue valorable. Se confirmó angiográficamente en todos, salvo en 2 pacientes, en que se practicó angio-RM en su lugar. No se han reflejado complicaciones neurológicas relacionadas con la angiografía. Además, se practicaron preoperatoriamente angio-TAC en $1 \mathrm{y}$ angio-RM en 11 pacientes (Figura 2).

El control postoperatorio fue con eco-Doppler en 83 casos, 15 con angio-RM, 1 con angiografía intraoperatoria, 1 con angio-TAC. Dos pacientes no han sido seguidos posteriormente.

Las diferencias en el grado de estenosis medidos mediante eco-Doppler y angiografía en 121 carótidas no fue estadísticamente significativa ( $p$ asociada a la $t$ de Student-Fisher para datos pareados de 0'27). Trece carótidas operadas presentaban una estenosis menor del 70\%, 22 entre 70 y $79 \%$, 38 entre 80 y $89 \%$, 24 entre 90 y $99 \%$.

Los pacientes presentaban preoperatoriamente en la TAC y/o RM craneal alteraciones ipsilaterales en 36 , contralaterales en 6, y múltiples en 24.

En relación con la situación de la carótida interna contralateral las complicaciones se valoran en la Tabla 4.

Dependiendo de su situación clínica y momento de la 
Tabla 3

Pacientes operados bilateralmente

\begin{tabular}{|c|c|c|c|c|c|c|}
\hline $1^{a}$ cirugía & $\%$ est & $2^{a}$ cirugía & $\%$ est & $\begin{array}{l}\text { Intervalo entre } \\
\text { cirugías }\end{array}$ & $\begin{array}{l}\text { complicaciones } \\
\text { postoperatorias }\end{array}$ & $\begin{array}{l}\text { complicaciones } \\
\text { tardías }\end{array}$ \\
\hline $14 / 11 / 95$ & $80-89$ & $9 / 12 / 96$ & $80-89$ & $390 \mathrm{~d}$ & $\begin{array}{l}\text { Desorientado tras } \\
2^{\mathrm{a}} \text { cirugía }\end{array}$ & \\
\hline 29/7/96 (CV) & - & $1 / 6 / 99$ & $80-89$ & $1036 \mathrm{~d}$ & & \\
\hline $17 / 3 / 97$ & $70-79$ & $21 / 4 / 97$ & $70-79$ & $34 \mathrm{~d}$ & $\begin{array}{l}\text { Infección urinaria } \\
\text { tras } 2^{\mathrm{a}} \text { cirugía }\end{array}$ & \\
\hline $15 / 6 / 98$ & $<70$ & $22 / 6 / 98$ & $<70$ & $7 \mathrm{~d}$ & $\begin{array}{l}\text { Reoperado por sangrado } \\
\text { en } 2^{\mathrm{a}} \text { cirugía }\end{array}$ & Pérdida visión ictal \\
\hline $24 / 11 / 98$ & $70-79$ & $30 / 11 / 98$ & $70-79$ & $6 \mathrm{~d}$ & & \\
\hline $9 / 2 / 99$ & $80-89$ & $2 / 3 / 99$ & $80-89$ & $21 \mathrm{~d}$ & $\begin{array}{l}\text { Desorientado tras } \\
2^{\mathrm{a}} \text { cirugía }\end{array}$ & \\
\hline 24/6/99 & $<70$ & 28/2/01 (CV) & $<70$ & $615 d$ & & \\
\hline $18 / 10 / 99$ & $70-79$ & $10 / 1 / 00$ & $70-79$ & $84 \mathrm{~d}$ & & \\
\hline $9 / 5 / 00$ & 90-99 & $24 / 7 / 00(\mathrm{CV})$ & $90-99$ & $76 \mathrm{~d}$ & & \\
\hline 13/12/01 (rr) & 100 & $7 / 2 / 02$ & $80-89$ & $54 \mathrm{~d}$ & & \\
\hline $15 / 1 / 02$ & $80-89$ & $21 / 2 / 02$ & $<70$ & $36 \mathrm{~d}$ & & \\
\hline
\end{tabular}

\% est: grado de estenosis de la arteria operada; CV: intervenciones realizadas por el servicio de Cirugía Vascular; rr: endarterectomía de carótida externa por obstrucción completa de la interna con relleno retrógrado.

cirugía, los pacientes se trataron con heparina intravenosa hasta el momento de la intervención, con heparina de bajo peso molecular o mediante antiagregantes (Tabla 5).

Se trataron con anestesia locorregional cuatro pacientes con obstrucción contralateral. Con edades entre 63 y 77 años, no presentaban síntomas establecidos al operarlos, salvo uno. En el postoperatorio, uno de ellos tuvo una afectación facial y otro una parálisis vocal que recuperaron completamente.

Cada paciente permaneció en el quirófano una mediana de 180 (rango 105-315) minutos, con un tiempo de clampaje de 20 (8-50) minutos y unas tensiones sistólica y diastólica durante el mismo de 150 (110-180) y 70 (40-100) $\mathrm{mm} \mathrm{Hg}$ respectivamente.

Diez pacientes pasaron a la unidad de recuperación post-anestésica o a la unidad de cuidados intensivos y luego a la planta ese mismo día. Los demás fueron trasladados a la planta al día siguiente, salvo en 14 ocasiones que requirieron varios días.

\section{Tabla 4}

Complicaciones según el estado de la arteria contralateral a la operada

\begin{tabular}{|l|c|c|c|c|}
\hline Contralateral & N ${ }^{\circ}$ cir & ComplNLpostop & ComplNLtardías & Exitus \\
\hline Oclusión & 11 & 1 & 0 & 1 \\
\hline Estenosis & 40 & 2 & 2 & 2 \\
\hline Normal & 46 & 1 & 6 & 1 \\
\hline Total & 97 & 4 & 6 & 4 \\
\hline
\end{tabular}

$N^{\circ}$ cir: Número de cirugías; ComplNLpostop: complicaciones neurológicas centrales en los 30 días tras la cirugía; ComplNLtardias: complicaciones neurológicas centrales tras los primeros 30 días de la cirugía; Exitus: exitus perioperatorios. 
Tabla 5

Tratamiento preoperatorio según situación clínica y complicaciones postquirúrgicas

\begin{tabular}{|l|c|c|c|c|c|c|c|c|}
\hline $\begin{array}{l}\text { Tratamiento } \\
\text { preoperatorio }\end{array}$ & Todos & $\begin{array}{l}\text { Estenosis } \\
\text { contralateral }\end{array}$ & $\begin{array}{l}\text { Obstrucción } \\
\text { contralateral }\end{array}$ & $\begin{array}{l}\text { Clínica establecida } \\
\text { al operarse }\end{array}$ & CoNL & Coex & Cohtma & Reint \\
\hline $\boldsymbol{a c}$ & 26 & 13 & 4 & 16 & 2 & 3 & 6 & 3 \\
\hline $\boldsymbol{h b}$ & 26 & 10 & 0 & 11 & 1 & 0 & 4 & 0 \\
\hline $\boldsymbol{a g}$ & 45 & 17 & 7 & 24 & 1 & 1 & 4 & 1 \\
\hline Total & 97 & 40 & 11 & 51 & 4 & 4 & 14 & 4 \\
\hline
\end{tabular}

$\boldsymbol{a c}$ : anticoagulación; $\boldsymbol{h b}$ : heparina de bajo peso molecular; ag: antiagregante; $\boldsymbol{C o N L}$ : complicaciones neurológicas centrales; $\boldsymbol{C o e x}$ : pacientes fallecidos en los 30 días de la cirugía; Cohtma: hematoma postquirúrgico; reint: pacientes reintervenidos en el postoperatorio inmediato.

Tabla 6

Pacientes fallecidos y con complicaciones neurológicas perioperatorias

\section{Exitus}

\begin{tabular}{|l|c|c|c|c|c|c|c|c|c|c|c|}
\hline & Sexo & Edad & Lado & \% Estenosis & Contralateral & Clínica & Can & rk & ASA & Imagen & Trto \\
\hline a & V & 78 & I & $90-99$ & Normal & Establecido & 25 & 3 & 3 & IPs & ac \\
\hline b & V & 76 & I & $80-89$ & Obstruida & Establecido & 95 & 1 & 2 & Mtp & ag \\
\hline c & V & 80 & D & $<70$ & Estenosis & Asintomático & 100 & 0 & 3 & Mtp & ac \\
\hline d & V & 74 & D & $80-89$ & Estenosis & Establecido & 85 & 2 & 3 & Mtp & ac \\
\hline
\end{tabular}

\section{Complicaciones neurológicas}

\begin{tabular}{|l|c|c|c|c|c|c|c|c|c|c|c|}
\hline & sexo & edad & Lado & \% Estenosis & Contralateral & Clínica & Can & rk & ASA & Imagen & Trto \\
\hline e & V & 76 & I & $80-89$ & Estenosis & Asintomático & 100 & 0 & 2 & No & hb \\
\hline f & V & 58 & I & $90-99$ & Obstruida & Establecido & 95 & 1 & 2 & Mtp & ac \\
\hline g & V & 77 & D & $<70$ & Estenosis & Asintomático & 100 & 0 & 2 & Ips & ag \\
\hline h & V & 68 & D & $80-89$ & Normal & Asintomático & 100 & 0 & 2 & Mtp & ac \\
\hline
\end{tabular}

Sexo V: varón; Lado operado I: Izquierdo, D: derecho; \% estenosis: grado de estenosis de la arteria operada; contralateral: situación de la arteria contralateral a la operada; Clínica: sin alteraciones neurológicas o con déficit establecido en el momento de la cirugía; gradación según escalas Can: escala neurológica canadiense; rk:escala de Rankin modificada; ASA: escala de gradación de riesgo anestésico de la American Society of Anesthesiologists; imagen preoperatoria por RM y/o TAC; Ips: lesiones isquémicas ipsilaterales a la carótida operada; Mtp: lesiones múltiples; Trto: tratamiento en el momento de la cirugía con: ac. Anticoagulación con heparina iv; $h b$ : heparina de bajo peso molecular; ag: antiagregante

Los pacientes ingresaron en nuestro Servicio desde la consulta de Neurología o fueron trasladados en el mismo ingreso desde la planta de Neurología. Estuvieron ingresados una mediana de 14 días, (rango 3-90), siendo la estancia postoperatoria de 4 días (rango 2-30). La cirugía se realizó a los 28 días (rango 2-605) del diagnóstico ultrasonográfico y a los 14 (1-455) días de la arteriografía. El último control por eco-Doppler tras la operación fue a los 52 (0-2832) días. Se revisaron clínicamente los pacientes 121 '5 (8-2106) días tras la cirugía. Los 12 fallecidos lo fueron a los 211'5 (2-1608) días de la intervención.

\section{Complicaciones perioperatorias}

Ocurrieron 4 exitus perioperatorios $\left(4{ }^{\prime} 4 \%\right.$ de pacientes, 4'1\% de cirugías) (Tabla 6)

a. Un paciente murió en su domicilio a los 19 días de la cirugía, por causas no determinadas. Tenía antecedentes de enfermedad pulmonar obstructiva crónica, fibrilación 
auricular y enfermedad vascular periférica.

$b$. Otro paciente falleció por disociación electromecánica cardíaca a los 2 días de su endarterectomía. Entre sus antecedentes constan diabetes, consumo de tabaco y alcohol e infarto cerebral 5 años antes, un probable infarto inferior detectado en el electrocardiograma sin clínica reflejada previamente.

c. Un tercer paciente falleció por neumonía 22 días después de ser operado. Fue tratado anteriormente por arritmia cardíaca. hipertensión arterial y enfermedad pulmonar crónica.

d. Finalmente, un paciente falleció por infarto cerebral masivo ipsilateral a los dos días de la intervención. Padeció previamente un infarto de miocardio.

Sucedieron 4 casos de empeoramiento neurológico postquirúrgico (4'4\% de pacientes, 4'1\% de cirugías) (Tabla 6)

e. Un paciente presenta postoperatoriamente una paresia leve en mano con posterior mejoría. Se encontraba en tratamiento por hipertensión arterial.

$f$. Otro paciente, con hemiparesia casi recuperada previamente a la cirugía, despierta hemipléjico, mejorando posteriormente. Su historia previa incluía tabaquismo e hipertensión arterial.

g. Un paciente sufrió un infarto de la cápsula interna debido a una disección carotídea. Su hemiparesia izquierda mejoró a largo plazo. Entre sus antecedentes, constan enfermedad vascular periférica, tabaquismo y episodios isquémicos transitorios repetidos.

$h$. Otro paciente, con parestesias en hemicara y miembro superior izquierdos, sufrió una paresia leve en el miembro superior izquierdo tras la intervención. Tenía antecedentes de hipertensión arterial, hiperlipidemia y tabaquismo.

En 4 pacientes (4,1\% de cirugías) hubo que reabrir la herida en el postoperatorio, uno en el mismo momento de despertarlo por hemiparesia (se realizó un estudio con contraste intraoperatorio que no mostró alteraciones evidentes, pero no se reabrió la arteria) y otro antes de despertarlo por sangrado abundante por drenaje (sin encontrar alteraciones significativas en la arteriotomía); otros dos pacientes, horas después, por hematoma local con compromiso de la vía respiratoria. Tres de ellos fueron tratados con anticoagulación hasta la cirugía y uno con antiagregantes. Un paciente con una neoplasia cervical operada y radiada requirió una traqueostomía por obstrucción de vía aérea a los 5 días de la endarterectomía, sin complicaciones a nivel de la herida.

Otros trastornos perioperatorios encontrados en estos pacientes fueron: fibrilación auricular en 2; infección urinaria en 2; una molestia dental achacada a la intubación, un cuadro febril no filiado y otro achacado a infección de vía central; crisis de artritis gotosa aguda en 1; neumonía en 2 (una fue causa del exitus); síndrome transitorio de secreción inadecuada de hormona antidiurética en 1; hipertensión arterial que requirió tratamiento específico en 7 e hipotensión arterial en otro. Un paciente reingresó por infección superficial de la herida y otro precisó atención en urgencias por un flemón dentario varios días tras su alta.

Se constataron 6 paresias faciales, una del hipogloso, y otra del nervio recurrente, todas transitorias. Hubo hematoma de la herida tras 14 cirugías, pero sólo 3 de los pacientes fueron reoperados debido a ello. De estos casos, 6 fueron tratados con anticoagulación hasta la cirugía, 4 con antiagregante, 4 con heparina de bajo peso molecular.

\section{Complicaciones postoperatorias a largo plazo}

7 pacientes (7'8\% de los pacientes) han fallecido posteriormente por otras causas (uno operado bilateralmente), 6 por cáncer a los 0'5-4 años de la cirugía y 1 por cardiopatía a los 3 años.

Se contabilizan 5 pacientes con nuevos episodios ictales a largo plazo, tres de ellos (3'3\% de los pacientes, 3'1\% de las intervenciones) en relación con la arteria operada:

-. Un paciente tuvo episodios repetidos de hemiparesia derecha al año de una endarterectomía izquierda. Se evidenció una estenosis izquierda del $70 \%$, pero no aceptó ser reintervenido, falleciendo por una neoplasia broncogénica a los 3 años de la cirugía.

-. Otro paciente presentó episodios bilaterales múltiples a los 4 años tras una cirugía derecha. Presentó una estenosis del $60 \%$ en la arteria operada. Esta situación se sigue de un deterioro general progresivo y severo que descarta la reintervención.

-. Se reflejó una hemianopsia ictal con pérdida de visión periférica en ojo derecho en un paciente con cirugía bilateral dos años antes, ambas carótidas estaban permeables.

-. Un ictus cerebeloso produjo ataxia residual en un paciente a los 5 años de una endarterectomía derecha, con control ultrasonográfico satisfactorio bilateralmente.

-. Otro paciente reingresó a los cuatro meses por un cuadro que cursó como un ictus protuberancial izquierdo transitorio sin estenosis significativa en la carótida derecha operada, pero con estenosis contralateral.

Se encontraron 7 estenosis en el control por imagen (7'2\% de cirugías). Un paciente, con obstrucción en el postoperatorio inmediato la mantenía a los dos meses. Una paciente operada por una seudooclusión angiográfica, presentó una oclusión en el control RM y Doppler, sin modificación de la clínica. Un paciente con estenosis del 70\%, 
con episodios ictales transitorios, prefirió no operarse; otro con estenosis del $60 \%$ y con clínica bilateral, sin problema cardíaco conocido, no se operó por su mal estado general; otros 3 pacientes con estenosis significativas permanecieron asintomáticos y no se reoperaron.

\section{Discusión}

\section{Morbimortalidad}

Hay varios estudios que demuestran la eficacia de la endarterectomía en mejorar la evolución natural del tratamiento médico de la enfermedad ateromatosa carotídea. En el estudio NASCET, el riesgo de ictus ipsilateral a los 3 años es del $2813 \%$ para los pacientes randomizados para tratamiento médico y del 8 ' $9 \%$ para los de tratamiento quirúrgico ${ }^{53}$. A los 11'9 meses de seguimiento en el "Veterans Affairs Study" 46 la frecuencia de ictus fue del 19'4\% en los no operados y del 7'7\% en los operados. En el ECST, la frecuencia estimada de un ictus mayor o muerte a los tres años fue del $26{ }^{\prime} 5 \%$ para el grupo control y $14 ' 9 \%$ para el grupo operado ${ }^{25}$. En ese mismo grupo ECST, el riesgo de ictus o muerte a los 3 años en pacientes con estenosis $70-99 \%$ en la arteria asintomática fue de $57^{\prime} \%^{24}$. El riesgo de ictus-muerte, tras endarterectomía, es un $40 \%$ menor en las estenosis asintomáticas que en las sintomáticas, de una manera consistente y generalizable ${ }^{60}$. Se consideran como límites aceptables de complicaciones perioperatorias alrededor del 6\% para pacientes sintomáticos ${ }^{16}$, del 3\% para los asintomáticos ${ }^{26,29}$, y del $10 \%$ para la enfermedad recurrente ${ }^{51}$. No obstante, las cifras de morbimortalidad son difíciles de comparar, pues oscilan ampliamente según los estudios y según quién realice el control clínico ${ }^{59}$.

Nuestra mortalidad y morbilidad neurológica central es del 8 '2\% de las cirugías. Es mayor que la generalmente referida en los estudios sobre pacientes sintomáticos (4'1$\left.7^{\prime} 5 \%\right)^{25,27,33,42,58,62}$ y especialmente en los asintomáticos $\left(2^{\prime} 3-5^{\prime} 4 \%\right)^{13,26,38,42,58}$. Esta elevada cifra tiene que ver, sobre todo, con la mortalidad de nuestra serie: hemos tenido 4 muertes perioperatorias ( $4^{\prime} 1 \%$ de las cirugías), mientras que las cifras reflejadas en la literatura oscilan entre $0 \mathrm{y}$ $2 \%$ y suelen ser más bajas que las de morbilidad neurológi$\mathrm{ca}^{26,27,38,51,54,56}$. Tres de los cuatro fallecidos en nuestro centro lo fueron por causas sistémicas no neurológicas, posiblemente en relación con su patología concomitante.

La cifra de complicaciones neurológicas (4'1\% de las cirugías), aunque alta, se compara mejor con las de otras series, que oscilan entre $9^{\prime} 9 \%^{14}$ y $00^{\prime} 7 \% \%^{47}$, incluido el $5 ' 2 \%$ del reciente estudio multicéntrico español ${ }^{56}$. En nuestra serie las complicaciones se distribuyen ampliamente durante los años y entre los cirujanos. Para el grupo de Rothwell, si la relación de exitus respecto a los ictus no fatales es significativamente superior a una relación $6 / 1$, deba tenerse en cuenta la posibilidad de que algunos ictus no fatales hayan pasado desapercibidos ${ }^{62}$. En nuestro caso, nos parece que el seguimiento ha sido adecuado en las primeras horas, que es cuando se producen la mayoría de las complicaciones $^{27,65}$, y casi todos los pacientes han podido ser controlados en el postoperatorio, al menos inicialmente. Sin embargo, el control postoperatorio no ha sido protocolizado a largo plazo ni con revisión externa, lo que con seguridad habría aumentado las cifras de complicaciones $^{59,62}$.

\section{Otras complicaciones}

Tuvimos en esta serie 4 reintervenciones (4'1\% de las cirugías), uno por déficit neurológico al despertar y tres por hematoma cervical. En el primero no se encontró oclusión o trombo. En 3 pacientes del estudio NASCET, reoperados por déficit inmediato no se encontraron anomalías significativas, pero en 9 con déficit diferido unas horas, había oclusión o trombo en todos ${ }^{27}$. La actual tendencia a no interrumpir la antiagregación ${ }^{23}$, y el uso de anticoagulación en los casos progresivos o de repetición, hace que la cifra de hematomas sea alta, pero sólo ha habido que intervenir una parte y en ningún caso hubo consecuencias graves como en otros estudios ${ }^{14,27}$. El número de hematomas operados es comparable a otras series ${ }^{13,14,27,56,58}$, aunque también se refieren cifras tan bajas como $0{ }^{\prime} 4 \%{ }^{44}$.

La infección de herida se reporta sólo ocasionalmente, nuestro $1 \%$ es un intermedio entre el $2 \%$ del estudio NASCET $^{27}$ y el deseable $0 \%$ del CASANOVA ${ }^{13}$. La incidencia de afectación de nervio periférico, $82 \%$, es comparable a algunos estudios cooperativos ${ }^{27}$, pero en ambos casos superior al $3-5^{\prime} 1 \%$ de otras series ${ }^{13,38,44,56,58}$. El evitar este tipo de morbilidad es una de las posibles ventajas de los procedimientos endovasculares ${ }^{14}$.

Diversas complicaciones médicas se produjeron en el $8^{\prime} 1 \%$ de los pacientes operados en el estudio NASCET ${ }^{52}$ y $45 \%$ en el CASANOVA ${ }^{13}$, siendo hasta tres veces superiores en los pacientes operados que en los tratados conservadoramente ${ }^{52}$. En nuestra serie han supuesto el $22{ }^{\prime} 6 \%$, en general transitorias y sin repercusión significativa para el paciente. Fueron variadas, en dos pacientes fatales, otras veces vagas, y en general difíciles de evaluar en relación con las alteraciones propias del enfermo y de los sucesivos procedimientos realizados. Creemos que la alta incidencia de comorbilidad en nuestros pacientes ha podido condicionar que uno de cada cinco pacientes precisara tratamiento específico de alguna patología preexistente o sobreañadida durante el postoperatorio. Se ha dicho que la tensión arterial se inestabiliza en 3/4 de los pacientes en las primeras horas y se producen hiper e hipotensiones que pueden asociarse a hemorragia o isquemia ${ }^{8}$. Uno de nuestros pacientes con hipertensión refractaria periope- 
ratoria requirió reintervención por hematoma y presentó postoperatoriamente crisis y un empeoramiento transitorio de su déficit previo.

\section{Resultados a largo plazo, nuevos ictus, estenosis и oclu- sión}

En nuestra serie, tras la primera revisión programada, las siguientes revisiones dependían de la evolución, y no siguieron un protocolo estricto, si bien los pacientes que empeoraron volvieron a ser revisados por los neurólogos o por nosotros, por lo que no podemos comparar nuestros resultados con los publicados en los estudios clásicos. Se acepta que los episodios isquémicos ocurren principalmente en los 2-3 años siguientes, tanto en los operados como en los no operados ${ }^{2,25}$. Contabilizamos en nuestro grupo nuevos episodios ictales en relación con la arteria operada en un 3'1\%, entre uno y 4 años después; y otros dos pacientes presentaron otra sintomatología isquémica a los 5 años y a los 4 meses. Las cifras publicadas en otros estudios prospectivos y con control externo son de alrededor del $30 \%$, aunque sólo son graves en su quinta parte ${ }^{27}$.

Tampoco hemos seguido un criterio específico de control por imagen tras el primer examen postoperatorio. Por el tipo de seguimiento que hemos realizado no es posible confirmarlo, pero parece que la frecuencia de reestenosis es mayor el primer año $(10 \%)$, decreciendo posteriormente hasta un $1 \%$ anual, y con un riesgo relativo de ictus entre 10 y $0^{\prime} 1^{32}$. Nosotros constatamos un $7^{\prime} 2 \%$ de estenosis en el último control de nuestros pacientes. No parece haber diferencia estadística en la incidencia de episodios transitorios, ictus o muerte entre pacientes con o sin lesiones recurrentes, que según la literatura tienen una incidencia entre el 10 y $23 \%{ }^{12}$. Por otro lado, se considera que el aumento de la estenosis no debe ser un criterio suficiente para operar a un paciente asintomático ${ }^{64}$. No obstante, los criterios de intervención, según la clínica y grado de estenosis, son similares a los de la primera cirugía, y el riesgo de complicaciones locales e isquémicas no tiene por qué ser mayor ${ }^{12,63}$. Así, no hemos reoperado ningún paciente con reestenosis asintomática. Dos de nuestros pacientes con estenosis tras la cirugía y clínica persistente no aceptaron o no fueron candidatos a reintervención. En el multicéntrico español la cifra de intervenciones por reestenosis es del $411 \%{ }^{56}$.

\section{Procedimientos}

Por su calidad de detalle, adecuada evaluación de la placa complicada y de la patología intracraneal asociada, aún consideramos la arteriografía el diagnóstico preoperatorio más adecuado ${ }^{29}$. En nuestro caso no ha habido complicaciones en relación con el procedimiento, aunque se ha publicado cifras entre $0{ }^{\prime} 4 \%^{38}$ y $1{ }^{\prime} 2 \%^{26}$. Con la mejora de los medios diagnósticos alternativos, probablemente en ciertos casos de alto riesgo no sea imprescindible $^{28,29,49}$ (Figura 2), sobre todo si se correlacionan bien los resultados de los diferentes estudios en cada centro, como es nuestro caso. En dos de nuestros pacientes se sustituyó por angio-RM satisfactoriamente. El control postoperatorio de nuestros pacientes ha sido no invasivo.

El tiempo de clampaje no parece estar asociado con una mayor incidencia de morbilidad neurológica postoperatoria en nuestra serie. Se asume en general que el origen de los síntomas neurológicos del territorio carotídeo es embó$\operatorname{lico}^{4,12}$, y se estima que los déficits postoperatorios suelen tener su origen en defectos de la técnica quirúrgica más que en los efectos hemodinámicos derivados del tiempo de clampaje, aunque este puede influir ${ }^{31}$. Por otro lado, hay que considerar que al menos un tercio de los ictus en estos pacientes se deben a patología cardioembólica o de otro tipos.

En un intento de minimizar las complicaciones de causa hemodinámica, se han preconizado múltiples estrategias. Por ejemplo, el empleo de shunt. Aún no hay evidencia científica suficiente en cuanto al beneficio de su uso', incluso parece que puede ser perjudicial ${ }^{20,31,56}$. Exige una exposición anatómica mayor, alarga la duración del procedimiento y complica su realización, por lo que en general no consideramos necesario su uso. Algunos autores prefieren la protección cerebral con barbitúricos a altas dosis, evitando el uso de shunt o de anestesia regional ${ }^{31}$, esto exige una estrecha monitorización anestésica y mediante EEG, que aún así es de limitado valor en esos casos ${ }^{4}$. Otros recomiendan asociar a estas medidas la expansión de volumen preoperatoria ${ }^{34}$. Tampoco existe evidencia clara del beneficio de la anestesia regional ${ }^{66}$. Esta no es un procedimiento estándar en nuestro medio, pero se ha empleado en cuatro de nuestros pacientes más recientes, con oclusión contralateral y de más edad que la media. En todos los casos al clampar la arteria disminuyó el nivel de conciencia del paciente, recuperándose si se desclampaba, y sin secuelas neurológicas posteriores. No hemos encontrado ventajas suficientes como para emplearla de manera habitual.

Hay una tendencia creciente a utilizar la monitorización perioperatoria intensiva, aunque no se ha correlacionado claramente con los resultados obtenidos ${ }^{31,59}$. Probablemente razones de marketing, legales $u$ otras tengan algo que ver en esta situación. En nuestro centro, no hemos dispuesto hasta recientemente de material y personal para el control neurológico intraoperatorio y aún no somos capaces de valorar su utilidad real.

El uso del microscopio es recomendado por algunos para una mayor precisión en la extirpación de la placa y sutura del vaso, sobre todo en las lesiones más proximales ${ }^{20}$. Nosotros hemos utilizado ocasionalmente este instrumento 
sin decidirnos claramente por su uso.

Se recomienda no revertir la heparina al finalizar la endarterectomía para prevenir la aparición inicial de trombos y esa es nuestra tendencia actual. Ello obliga a una hemostasia más cuidadosa, sin aumentar necesariamente la cifra de hematomas postquirúrgicos ${ }^{20}$.

Se recomienda con frecuencia el uso de parche para el cierre arterial. Parece disminuir el riesgo de oclusión arterial y reestenosis, pero no está claro si reduce el riesgo de muerte o ictus ${ }^{17,33}$. Nos parece que alarga el procedimiento y puede dar lugar a complicaciones añadidas propias. Pensamos que es más importante que su uso el tratamiento adecuado de la placa y de la pared residual y la posterior y persistente educación del paciente en el control de factores de riesgo ateromatoso. Sin embargo, puede ser necesario cuando, por problemas técnicos o anatómicos, la arteria no puede cerrarse sin estenosis significativa.

\section{Análisis según posibles factores de riesgo}

Aunque la edad ed,54 $^{25}$ el $\operatorname{sexo}^{27,39}$ no parecen influir en la mortalidad, todos nuestros fallecidos fueron mayores de 74 años y varones. En nuestra serie ninguna mujer (11'1\% de los pacientes operados, 10'3\% de las cirugías) tiene mortalidad ni morbilidad neurológica perioperatoria ni en el seguimiento posterior. Una presenta hipertensión postoperatoria y dos hematomas en herida, no significativos. En otras series se ha encontrado mayor riesgo quirúrgico entre las mujeres ${ }^{25,33}$.

No hemos considerado, a la hora del tratamiento quirúrgico, la patología intracraneal oclusiva asociada, que puede ${ }^{27}$ asociarse o no tener relación con una mayor morbimortalidad $^{12}$.

Se ha demostrado una relación inversa de los resultados con el número de intervenciones ${ }^{42,54,56}$, y algunos recomiendan un número mínimo anual de al menos 6 cirugías $^{42}$. $\mathrm{O}^{\prime} \mathrm{Neill}^{51}$ niega esta relación a partir de un mínimo bianual de 3 cirugías, pero encuentra influyentes la especialidad y el tiempo de práctica del cirujano: halló una mayor morbilidad entre los cirujanos recientemente licenciados y una mortalidad menor entre los neurocirujanos. La necesidad de aprendizaje se confirma en un estudio realizado por dos cirujanos ${ }^{58}$, en que la morbimortalidad de los primeros 50 casos fue del $7 \%$ y la de los siguientes $50 \mathrm{del}$ $4 \%$. En un reciente estudio en EEUU en el año 2000, los neurocirujanos practicaban 5'7 (rango 1-54) endarterectomías al año mientras que la cifra para cirujanos de otras especialidades era de 11' 1 (rango 1-154) ${ }^{18}$. En nuestro caso la mediana de cirugías al año por cirujano es de 3, (rango 0-10). La necesidad de un número mínimo de cirugías para garantizar unos resultados aceptables no es un problema fácil de solucionar, pues según Rothwell ${ }^{59}$, para demostrarse una competencia suficiente en endarterectomías en pacientes sintomáticos un cirujano debería realizar al menos 150 procedimientos.

La estenosis contralateral, puede ser un factor de riesgo ${ }^{1,21,27,31,55,56}$, aunque no siempre contraindica la indicación quirúrgica $^{12}$. La proporción de nuestros pacientes con obstrucción contralateral (11'3\%) es algo mayor que la de otros estudios ${ }^{62}$. Doce de los 19 pacientes con riesgo potencial de ictus en la serie de 37 pacientes de Frawley tenían estenosis $>70 \%$ u oclusión contralateral, aunque ninguno tuvo un déficit postoperartorio ${ }^{31}$. En nuestro estudio, ocurrió un déficit neurológico postoperatorio en el 9\% de los pacientes intervenidos con obstrucción contralateral, en el $5 \%$ de los que tenían estenosis contralateral, y en el $2 \%$ de los que no presentaban estenosis contralateral significativa.

Con los resultados que disponemos, hay que ser cautos a la hora de la indicación quirúrgica en enfermos con estenosis carotídea. Parece que la cirugía es contraproducente para pacientes con menos del $30 \%$ de estenosis y no beneficiosa para aquellos con estenosis $30-49 \%$ o casi ocluidos ${ }^{16,62}$, no todos los pacientes con una estenosis sintomática mayor del 70\% se benefician necesariamente de la cirugía ${ }^{48,61}$, y la reducción de riesgo absoluto es mínima en la cirugía de pacientes asintomáticos ${ }^{15}$. En efecto, uno de nuestros pacientes casi ocluidos no mejoró su sintomatología ni su imagen con la cirugía.

Hemos tratado más pacientes con estenosis grave que en algunos estudios clásicos. En nuestra serie 13 carótidas operadas presentaban una estenosis $<70 \%$ y 84 una estenosis entre 70 y 99\%. La morbimortalidad fue de 2 (15'4\%) y $6\left(7^{\prime} 1 \%\right)$ casos respectivamente. También en el NASCET ${ }^{27}$ la morbimortalidad a los 30 días fue mayor en los 1087 pacientes con estenosis moderada (6'7\%) que en los 328 con estenosis graves $\left(5^{\prime} 8 \%\right)$. En la reciente revisión del ECST con mediciones adaptadas a las de NASCET, las cifras fueron de 7' $8 \%$ para los 1415 pacientes con estenosis menores del 70\% y 6'1\% para los 327 pacientes con más del $70 \%$ o seudooclusiones ${ }^{62}$.

La edad de nuestros pacientes, 69 años de mediana, es levemente más alta que los 62'5-67 años de estudios cooperativos $^{14,25,26,50}$. Setenta de nuestros pacientes tenían 65 años o más cuando fueron operados y 9 de ellos 80-86 años. Actualmente se tiende a aceptar como candidatos quirúrgicos a pacientes mayores ${ }^{37,54,55}$, en los que los riesgos son más elevados ${ }^{3}$ pero también el posible beneficio es más significativo $^{2}$. Algunos dicen que los pacientes ancianos aumentan su estancia postoperatoria por complicaciones menores $^{54}$, pero en nuestros pacientes de 80 o más años, su estancia ha sido 3 días (rango 2-22), similar a los 4 (rango 2-30) de toda la serie. Además, de los 8 pacientes que presentaron morbimortalidad, siete eran menores de 80 años, y otras morbilidades tampoco fueron especialmente asociadas con la edad, por lo que adoptamos para los pacientes 
añosos los mismos criterios que para los demás pacientes.

Actualmente se cree que la cirugía precoz puede ser beneficiosa ${ }^{25}$ evitando el riesgo de ictus recurrentes si se sigue el criterio arbitrario de esperar 6 semanas tras la clínica inicial. La endarterectomía puede realizarse con relativa seguridad en los 15 días siguientes a un ictus agudo no incapacitante ${ }^{57}$, y no se recomienda postponerla más de 30 días tras un infarto hemisférico no invalidante con estenosis mayor del $70 \%{ }^{29}$. Por otro lado, no se deben operar pacientes con déficits de más de dos horas de evolución u oclusiones carotídeas agudas de más de seis horas de evolución ${ }^{21}$. Como los pacientes con accidentes isquémicos transitorios "in crescendo" parecen ser los de peor pronóstico y no hay diferencias en el número de ictus entre cirugía precoz y tardía ${ }^{10,33}$, hemos acelerado la cirugía en lo posible en los pacientes con síntomas de repetición, pero sin realizar cirugías de urgencia. En los pacientes estables, el momento de la cirugía dependió en parte de las disponibilidades de quirófano, de la situación clínica y del deseo del paciente.

\section{En fin, ¿Hacemos las cosas bien?}

Nuestros resultados son claramente mejorables. El poner en números nuestra actuación frecuentemente nos sorprende y siempre aclara las cosas. Como recomienda el grupo de Reina ${ }^{56}$, es bueno conocer nuestras cifras reales y saber qué podemos ofrecer a nuestros pacientes, lo que, por desgracia, no suele coincidir con los excelentes resultados obtenidos por buenos especialistas en centros de calidad. Las causas reales de las complicaciones de los pacientes no suelen ser evidentes. En general parecen estar relacionadas con problemas técnicos y las características del paciente. Los primeros dependen de la experiencia y volumen de intervenciones del cirujano ${ }^{42,51,54,56}$, pero aún en manos hábiles, cada paciente es distinto y el reto técnico y las dificultades intraoperatorias no son excepcionales ${ }^{36}$. Es fundamental centrarse en lo que se debe hacer; primero elegir al paciente adecuado que debe ser intervenido y no recomendar la cirugía a quien no pueda beneficiarse de ella; luego, eliminar la placa de una manera completa y sin causar daño al paciente, siendo el tipo de técnica y aparataje empleados de una importancia secundaria si se consigue este fin. Es difícil saber qué pacientes pueden beneficiarse de determinadas técnicas (shunt, barbitúricos, parche... ) pues los resultados dependen en gran medida del que los aplica ${ }^{31}$, pero quizás sea recomendable evitar posturas rígidas. Una vez valorada la indicación quirúrgica en conjunción con los neurólogos, no hemos rechazado ningún paciente que quisiera operarse a pesar de su edad o de su situación clínica. Así la mitad de ellos tenían un grado ASA III o IV y 51 tenían algún déficit neurológico establecido en el momento de la intervención. Si seleccionamos mejor los pacientes ${ }^{10,21,43,61}$, probablemente los resultados generales sean mejores, pero podemos privar a alguno de un posible beneficio ${ }^{6}$. En cualquier caso, es necesario una técnica brillante y un control adecuado de las comorbilidades para obtener el mejor resultado posible.

\section{Conclusión}

La endarterectomía carotídea es una técnica quirúrgica establecida en el tratamiento de pacientes que han sufrido un evento neurológico en relación con estenosis de al menos el $70 \%$ de la luz arterial en la carótida interna cervical. La indicación es más discutida y en cualquier caso debe ser individualizada en los pacientes con estenosis de alto grado pero asintomáticos.

En dicha situación además de la habilidad del cirujano, los factores clínicos y radiológicos de cada individuo juegan un papel importante en la toma de decisión. Las estenosis de bajo grado evolucionan mejor con tratamiento conservador.

Es evidente que se trata de pacientes con alto riesgo quirúrgico, debido a la concurrencia de múltiples enfermedades asociadas, por lo que las cifras de morbimortalidad de cada equipo quirúrgico son relevantes, y los resultados deben, en todo caso, superar las expectativas de la historia natural del proceso. Es aconsejable una monitorización permanente de los resultados en estos pacientes.

Esta es una enfermedad un tanto olvidada por los Servicios de Neurocirugía de nuestro medio que creemos necesario retomar dada la complejidad del manejo perioperatorio, esencialmente debida a la referida comorbilidad, que no se limita a la patología local.

A la vista de la modesta casuística expuesta, creemos factible realizar endarterectomías carotídeas en centros con un número teóricamente bajo de caso anuales, seleccionando correctamente los pacientes, depurando la técnica y estudiando con rigor los factores que se asocian de forma individual con un riesgo aumentado de complicaciones. La revisión sistemática de nuestros resultados es un arma más en nuestro permanente deseo de mejora.

\section{Agradecimientos}

Fernando Sanjuán, Eduardo Rossi, Francisco GómezTaborga, José María Borrás participaron en el tratamiento de estos pacientes y José María Trejo nos aconsejó en la redacción del manuscrito.

\section{Bibliografía}

1. AbaRahma, A.F., Metz, NI., Robinson, P.A.: Natural history of $>$ or $=60 \%$ asymptomatic carotid stenosis in patients with contralateral carotid occlusion. Ann Surg 2003; 
238: 551-561.

2. Alamowitch, S., Eliasziw, M., Algra, A., Meldrum, H., Barnett, H.J.M.: Risk, causes, and prevention of ischaemic stroke in elderly patients with symptomatic internal-carotidartery stenosis. Lancet 2001; 357: 1154-1160.

3. Alozairi, O., MacKenzie, R.K., Morgan, R., Cooper, G., Engeset, J., Brittenden, J.: Carotid endarterectomy in patients aged 75 and over: early results and late outcome. Eur J Vasc Endovasc Surg 2003; 26: 245-249.

4. Arnold, M., Sturzenegger, M., Schaffler, L., Seller, R. W.: Continuous intraoperative monitoring of middle cerebral artery blood flow velocities and electroencephalography during carotid endarterectomy. A comparison of the two methods to detect cerebal ischemia. Stroke 1997; 28: 13451350 .

5. Barnett, H.J.M., Gunton, R.W., Eliasziw, M., et al.: Causes and severity of ischemic stroke in patients with internal carotid artery stenosis. JAMA 2000, 283: 1429-1436.

6. Barth, A., Bassetti, C.: Patient selection for carotid endarterectomy: How far is risk modeling applicable to the individual? Stroke 2003; 34: 524-527.

7. Benavente, O., Moher, D., Pham, B.: Carotid endarterectomy for asymptomatic carotid stenosis: a meta-analysis. BMJ 1998; 317: 1477-1480.

8. Biller, J., Feinberg, W.M., Castaldo, J.E., et al.: Guidelines for carotid endarterectomy. A statement for healthcare professionals from a special writing group of the Stroke Council, American Heart Association. Circulation 1998; 97: 501509.

9. Bond, R., Rerkasem, K., Rothwell, P.M.: Routine or selective carotid artery shunting for carotid endarterectomy (and different methods of monitoring in selective shunting) (Cochrane Review). The Cochrane Library, Issue 12003. Oxford: Update Software.

10. Bond, R., Rerkasem, K., Rothwell, P.M.: Systematic review of the risks of carotid endarterectomy in relation to the clinical indication for and timing of surgery. Stroke 2003; 34 : 2290-2301.

11. Borrás, J.M., Peerless, S. J.: Manejo actual de la estenosis carotídea. ¿Qué es lo que la evidencia nos demuestra? Rev neurol 1999. 28: 256-263.

12. Callow, A.D.: Surgery of the carotid and vertebral arteries for the prevention of stroke. Baltimore. Williams \& Wilkins, 1996.

13. CASANOVA Study Group. Carotid surgery versus medical therapy in asymptomatic carotid stenosis. Stroke 1991; 22: 1229-1235.

14. CAVATAS investigators. Endovascular versus surgical treatment in patients with carotid stenosis in the Carotid and Vertebral Artery Transluminal Angioplasty Study (CAVATAS): a randomised trial. Lancet 2001; 357: 1729-1737.

15. Chambers, B.R., You, R.X., Donnan, G.A.: Carotid endarterectomy for asymptomatic carotid stenosis (Cochrane Review). The Cochrane Library, Issue 1 2003. Oxford: Update
Software.

16. Cina, C. S., Clase, C.M., Haynes, R.B.: Carotid endarterectomy for symptomatic carotid stenosis (Cochrane Review). The Cochrane Library, Issue 1 2003. Oxford: Update Software.

17. Counsell, C., Salinas, R., Warlow, C., Naylor, R.: Patch angioplasty versus primary closure for carotid endarterectomy (Cochrane Review). The Cochrane Library, Issue 12003. Oxford: Update Software.

18. Cowan, J.A., Wainess, R., Dimick, J.B., Upchurch, GR., Thompson, B.G.: Utilization of carotid endarterectomy by neurosurgeons in the United States from 1990 to 2000. Neurosurgery 2003; 53486 (Abstract).

19. Crawley, F., Brown, M.M.: Percutaneous transluminal angioplasty and stenting for carotid artery stenosis (Cochrane Review). The Cochrane Library, Issue 1 2003. Oxford: Update Software.

20. Crowell, R.M., Ogilvy, C.S., Ojeman, R.G.: Extracranial carotid artery atherosclerosis; carotid endarterectomy. En Wilkins RH and Rengachary SS (Eds). Neurosurgery. Nueva York; McGraw Hill, 1996; pp 2103-2115.

21. Delgado Bona, G., Gallego Culleré, J., Gil-Peralta, A, González Marcos, J.R.: Endarterectomia y angioplastia transtuminal percutánea carotídea en la prevención del infarto cerebral. Rev Clin Esp 1996; 196: 46-50.

22. Doménech, J.M.: Comparación de dos medias: pruebas t. En Fundamentos de diseño y estadística. Barcelona, Signo, 2002.

23. Engelter, S., Lyrer, P.: Antiplatelet therapy for preventing stroke and other vascular events after carotid endarterectomy (Cochrane Review). The Cochrane Library, Issue 1 2003. Oxford: Update Software.

24.European Carotid Surgery Trialist Collaborative Group. Risk of stroke in the distribution of an asymptomatic carotid artery. Lancet 1995; 345: 209-212.

25. European Carotid Surgery Trialist' Collaborative Group. Randomised trial of endarterectomy for recently symptomatic carotid stenosis: final results of the MRC European Carotid Surgery Trial (ECST). Lancet 1998; 351: 13791387.

26. Executive Committee for the Asymptomatic Carotid Atherosclerosis Study. Endarterectomy for asymptomatic carotid artery stenosis. JAMA 1995; 273: 1421-1428.

27. Ferguson, G.G., Eliasziw, M., Barr, H.W.K., et al.: The North American Symptomatic Carotid Endarterectomy Trial. Surgical results in 1415 patients. Stroke 1999; 30: 17511758.

28. Fernández, V., Bellmunt, S., Escribano, J.M., et al.: Indicaciones de endarterectomía carotídea sin arteriografía. Estudio de validación mediante eco-Doppler. Rev Neurol 2000; 31: 412-416.

29. Findlay, J.M., Tucker, W.S., Ferguson, G.G., Holness, R.O., Wallace, M.C., Wong, J.H.: Guidelines for the use of 
carotid endarterectomy: current recommendations from the Canadian Neurosurgical Society. Can Med Assoc J 1997; 157 : 653-659.

30. Forteza, A., Rabinstein, A.: Angioplastia y stenting de la estenosis carotídea. ¿Alternativa terapeútica o posibilidad técnica? Rev Neuro12001; 32: 270-275.

31. Frawley, J.E., Hicks, R.G., Beaudoin, M., Woodey, R.: Hemodynamic ischemic stroke during carotid endaterectomy: An appraisal of risk and cerebral protection. J Vasc Surg 1997; 25: 611-619.

32. Frericks, H., Kievit, J., van Baalen, J.M., van Bockel, J.H.: Carotid recurrent stenosis and risk of ipsilateral stroke. Asystematic review of the literature. Stroke 1998; 29: 244250 .

33. Golledge, J., Cuming, R., Beattie, D.K., Davies, A.H., Greenhalgh, R.M.: Influence of patient-related variables on the outcome of carotid endarterectomy. J Vasc Surg 1996; 24 : 120-126.

34. Gross, C.E., Bednar, M.M., Lew, S.M., Florman, J.E., Kohut, J.J.: Preoperative volume expansion improves tolerance to carotid artery cross-clamping during endarterectomy. Neurosurgery 1998; 43: 222-228.

35. Halm, E.A., Chassin, M.R., Tuhrim, S., et al.: Revisiting the appropriateness of carotid endarterectomy. Stroke 2003; 34: 1464-1471.

36. Heros, R.C.: La endarterectomía y la angioplastia carotídea: perspectiva quirúrgica. Rev Neurol 2001; 32: 266269.

37. Hoballah, J.J.: Carotid endarterectomy in elderly patients. En Loftus CM y Kresowik TF (Eds). Carotid Artery Surgery. New York; Thieme Medical Publishers Inc., 2000; pp187-195

38. Hobson, R.W., Weiss, D.G., Fields, W.S., et al.: Efficacy of carotid endarterectomy for asymptomatic carotid stenosis. N Eng1 J Med 1993; 328: 221-227.

39. Kapral, M.K., Wang, H., Austin, P. C., et al.: Sex differences in carotid endarterectomy outcomes: results from the Ontario Carotid Endarterectomy Registry. Stroke 2003; 34: 1120-1125.

40. Kilaru, S., Korn, P., Kasirajan, K., et al.: Is carotid angioplasty and stenting more cost effective than carotid endarterectomy? J Vasc Surg 2003; 37: 331-339.

41. Kresowik, T.F., Bratzler, D., Karp, HA., et al.: Multistate utilization, processes, and outcomes of carotid endarterectomy. J Vasc Surg 2001; 33: 227-235.

42. Kucey, D.S., Bowyer, B., Iron, K., Austin, P., Anderson, G., Tu, J.V.: Determinants of outcome after carotid endarterectomy. J Vasc Surg 1998; 28: 1051-1058.

43. Kuhan, G., Gardiner, E.D., Abidia, A.F., et al.: Risk modellig study for carotid endarterectomy. Br J Surg 2001; 88: 1590-1594.

44. Loftus, C.M.: Extracranial carotid lesions that should be managed with angioplasty and stenting. Clin Neurosurg
2005; 16: 93-107

2000; 47: 249-256

45. Loftus, C.M., Stanfield, MR.: Carotid endarterectomy. En Batjer HH y Loftus CM (Eds). Textbook of Neurological Surgery. Hagestown, USA; Lippincot Williams and Willdns, 2002; pp 2259-2269.

46. Mayberg, M.R., Wilson, S.E., Yatsu, F., et al.: Carotid endarterectomy and prevention of cerebral ischemia in symptomatic carotid stenosis. Veterans Affairs Cooperative Studies Program 309 Trialist Group. JAMA 1991, 266: 3289-3294.

47. Mullenix, P. S., Andersen, C.A., Olsen, S.B., Tollefson, D.F.: Carotid endarterectomy remains the gold standard. Am J Surg 2002; 183: 580-583.

48. Naylor, A.R., Rothwell, P.M., Bell, P.R.: Overview of the principal results and secondary analyses from the European and North American randomised trials of endarterectomy for symptomatic carotid stenosis. Eur J Vasc Endovasc Surg 2003; 26: 115-29.

49. Nederkoom, P.J., van der Graaf, Y., Hunink, M.G.: Duplex ultrasound and magnetic resonante angiography compared with digital subtraction angiography in carotid artery stenosis: a systematic review. Stroke 2003; 34: 1324-1332.

50. North American Symptomatic Carotid Endarterectomy Trial Collaborators.: Beneficial effect of carotid endarterectomy in symptomatic patients with high-grade carotid stenosis. N Engl J Med 1991; 325: 445-453.

51. O'Neill, L., Lanska, D.J., Hartz, A.: Surgeon characteristics associated with mortality and morbidity following carotid endarterectomy. Neurology 2000; 55: 773-781.

52. Paciaroni, M., Eliasziw, M., Kappelle L.J., et al.: Medical complications associated with carotid endarterectomy. Stroke 1999; 30: 1759-1763.

53. Paciaroni, M., Eliasziw, M., Sharpe, B.L., et al.: Longterm clinical and angiographic outcomes in symptomatic patients with $70 \%$ to $99 \%$ carotid artery stenosis. Stroke 2000; 31: 2037-2042.

54. Perler, B.A., Dardik, A., Burleyson, G.P., Gordon T.A., Williams, G.M.: Influence of age and hospital volume on the results of carotid endarterectomy: a statewide analysis of 9918 cases. J Vasc Surg 1998; 27: 25-33.

55. Reed, A.B., Gaccione, P., Belkin, M., et al.: Preoperative risk factors for carotid endarterectomy: defining the patient at high risk. J Vasc Surg 2003; 37: 1191-1199.

56. Reina-Gutiérrez, T., Arribas-Díaz, A., MasegosaMedina, A., Porto-Rodríguez, J., Serrano-Herrando, F.J.: Control de calidad en cirugía carotídea. Resultados del registro de cirugía carotídea en Región Centro de España (1999-2000). Rev Neurol 2003; 36: 9-14.

57. Ricco, J.B., Illuminati, G., Bouin-Pineau, M.H., et al.: Early carotid endarterectomy after a nondisabling stroke: a prospective study. Ann Vasc Surg 2000; 14: 89-94.

58. Ross, I.B., Guzmán, R.P.: Carotid endarterectomy results in the early years of practice. Surg Neurol 2001; 56: 46-51. 
59. Rothwell, P.M., Slattery, J., Warlow, C.P.: A systematic review of the risks of stroke and death due to endarterectomy for symptomatic carotid stenosis. Stroke 1996; 27: 260-265.

60. Rothwell, P.M., Slattery, J., Warlow, C.P.: A systematic comparison of the risks of stroke and death due to Carotid endarterectomy fos symtomatic and asymtpomatic stenosis. Stroke 1996; 27: 266-269.

61. Rothwell, P.M., Warlow, C.P.: Prediction of benefit from carotid endarterectomy in individual patientes: a riskmodelling study. Lancet 1999; 353: 2105-2110.

62. Rothwell, P.M., Gutnikov, S.A., Warlow, C.P.: Reanalysis of the final results of the European Carotid Surgery Trial. Stroke 2003, 34: 514-523.

63. Sharp, W.J.: Recurrent carotid artery stenosis: risk factors, prevention and treatment. En Loftus CM y Kresowik TF (Eds). Carotid Artery Surgery. New York; Thieme Medical Publishers Inc., 2000; pp 501-510.

64. Sleight, S.P., Poloniecki, J., Halliday, A. W.: Asymptomatic carotid stenosis in patients on medical treeatment alone. Eur J Vasc Endovasc Surg 2002; 23: 519-523.

\section{Comentario al trabajo: Morbimortalidad en endarterec- tomía carotídea de J.M. Castilla y cols.}

Los autores comunican su experiencia en 90 pacientes consecutivos sometidos a una endarterectomía carotídea en un hospital con bajo volumen de pacientes. Los resultados de un 4 por ciento de mortalidad y del 4 por ciento de ictus postoperatorios son globalmente aceptables e indican que la endarterectomía se puede realizar con garantías en un centro con bajo volumen de pacientes, siempre que se sigan los criterios de los autores en cuanto a correcta selección y empleo de una depurada técnica o puesto en palabras de los propios autores: "primero elegir al paciente adecuado que debe ser intervenido y no recomendar la cirugía a quien no pueda beneficiarse de ella; luego, eliminar la placa de una manera completa y sin causar daño al paciente ...... quizás sea recomendable evitar posturas rígidas". Estoy de acuerdo con el anterior párrafo y creo que los autores han hecho un buen trabajo que debe animarnos a continuar por esta senda quirúrgica que, salvo excepciones, ha sido abandonada por los neurocirujanos de este país.

En cuanto al manuscrito en si, creo que está bien elaborado, con información suficiente y con una buena discusión y bibliografía. Solamente creo que deberían revisar la redacción de los resultados para hacerlos un poco mas comprensibles.

La técnica descrita es la aceptada y añadiría un par de comentarios:
65. Tan, K.T., Cleveland, TJ., Berczi, V., McKevitt, F.M., Venables, G.S., Gaines, P.A.: Timing and frequency of complications after Caotid artery stenting: what is the optimal period of observation? J Vasc Surg 2003, 38: 23643.

66. Tangkanakul, C., Counsell, C., Warlow, C.: Local versus general anaesthesia for carotid endarterectomy). (Cochrane Review). The Cochrane Library, Issue 12003. Oxford: Update Software..

67. Winslow, G.M., Solomon, D.H., Chassin, M.R., Koscoff J., Merrick, N.J., Brook, R.H.: The appropriateness of Carotid endarterectoiny. N Engl J Med 1988; 318: 721-727.

Castilla, J.M.; Delgado, P.; Martín, V.; Fernández-Arconada, O,; Martín-Santidrián, M.A.; Rodríguez-Salazar. A.: Morbimortalidad en la endarterectomía carotídea. Neurocirugía 2005; 16: 93-107.

Correspondencia postal: José Manuel Castilla. Servicio de Neurocirugía. Hospital "General Yagüe". Avda. del Cid 96. 09005 Burgos.

- En primer lugar, me llama la atención que no mencionen el uso del microscopio para la realización de la endarterectomía y sutura de la herida. Nosotros hacemos uso habitual del microscopio y supone, como en otras cirugías, un importante avance por razones evidentes.

- En segundo lugar nosotros empleamos sistemáticamente métodos de monitorización encefálica en forma de EEG intraoperatorio $\mathrm{y}$, inicialmente, DOPPLER transcraneal. Ha habido pacientes que han mostrado depresión del registro EEG y en los que con elevación de la TA se han recuperado. El DOPPLER transcraneal es algo engorroso y no guardaba una relación con los hallazgos de la EEG, por lo que decidimos dejar de usarlo. Es de destacar que no hemos precisado colocar ningún shunt incluso en los pacientes con oclusión de la carótida contralateral. No hemos tenido déficits isquémicos postoperatorios en relación con el clampaje.

- En tercer lugar, me llama la atención el hecho de que los autores reviertan la heparina. Creo que no es necesario y que añade un riesgo de trombosis innecesario. Nosotros no revertimos la heparina $y$, tras un periodo inicial en que sí lo hacíamos, tampoco suspendemos la medicación antiagregante de los pacientes. No hemos encontrado mayor incidencia de complicaciones hemorrágicas.

J.M. Cabezudo Artero Badajoz 